\title{
The Gran Sasso Laboratory and Physics Programme
}

\author{
E. Bellotti \\ Università degli Studi di Milano \\ Laboratorio Nazionale del Gran Sasso - I.N.F.N.
}

An Associate Member of EPS

In the 1970's, after the success of the electroweak theory, several Grand Unification Models for the strong and electroweak interactions were proposed. They predicted proton decay with a half-life of $10^{30}$ years or less, and many other phenomena forbidden by the Standard Model of elementary particles. Experiments on proton decay started in Europe, USA, Japan and India and several substantial detectors were installed in mines or road tunnels. However, the need for a new, large, well equipped underground laboratory was clear.

A. Zichichi, who was at the time President of I.N.F.N., the Italian national agency for basic nuclear and subnuclear research, proposed to the Italian government the construction of the Laboratorio Nazionali del Gran Sasso (LNGS). The project was approved and two laws, in February 1982 and June 1984, allocated some 80000 million lire. The Laboratory is now in operation and the first results on a variety of subjects have been obtained.

\section{FACILITIES}

The Gran Sasso Massif, the highest mountain in the Apennines, is pierced by a pair of $10.4 \mathrm{~km}$ long tunnels on the highway from Rome to Teramo by the Adriatic Sea. At $6.4 \mathrm{~km}$ from the west entrance, close to the village of Assergi (L'Aquila), a by-pass leads into the Laboratory. The Laboratory consists of three large halls - designated A, B and $\mathrm{C}$ - service and transit areas, safety tunnels and, to the north, three small tunnels dedicated to geophysics. The total excavated volume is $\approx 180000 \mathrm{~m}^{3}$ and the total surface area is $\approx 15000 \mathrm{~m}^{2}$.

A few buildings not far from the west entrance host small laboratories, a mechanical workshop, and offices for physicists and administrative personnel.

Professor Enrico Bellotti is the Director of the Laboratorio Nazionale del Gran Sasso, I-67010 Assergi $(A Q)$. He is also with the Dipartimento di Fisica dell'Università di Milano, Via G. Celoria 16, I-20133 Milano.
The geographical position of the laboratory is very favorable: Gran Sasso is easily reached, being linked to the European network of highways, thus facilitating the transportation and installation of heavy equipment.

The general facilities (venting system, safety system, etc.) will not be discussed here; let me only mention data transmission which received special attention. A cable containing 100 optical fibres guarantees a very large transmission rate (some hundreds of Mbit/s) between underground laboratories and the external buildings and then on to standard links with national and international networks.

The rock overburden averages about $1400 \mathrm{~m}$ while the minimum thickness is $\approx 1000 \mathrm{~m}$. The energy threshold (for muons) is therefore $\geq 1 \mathrm{TeV}$ and the muon flux is reduced to $<1$ particle per $\mathrm{m}^{2} \mathrm{~h}$ (compared with a flux of $\approx$ $5 \times 10^{5}$ muons per $\mathrm{m}^{2} \mathrm{~h}$ at the surface). Thanks to the nature of the rock, local radioactivity is quite low. For instance, the thermal neutron flux is only $10^{-6}$ neutrons $/\left(\mathrm{m}^{2} \mathrm{~s}\right)$.

\section{PHYSICS PROGRAMME}

The Laboratory is dedicated mainly to subnuclear physics and to basic problems in astrophysics. However, owing to its special location a few experiments in geophysics, which will be not discussed, are also in progress.

\section{Double Beta Decay}

The term "double beta decay" (or d.b.d.) indicates one of the following processes:
1) $(A, Z) \rightarrow(A, Z+2)+2 e^{-}+2 \bar{v}$
II) $(A, Z) \rightarrow(A, Z+2)+2 e$
III) $(A, Z) \rightarrow(A, Z+2)+2 e^{-}+\chi$

(or similar processes with either positron emission or electron capture), where $A$ and $Z$ are respectively the atomic weight and the atomic number of an even-even nucleus, i.e. a nucleus having an even number of protons and neutrons. $\chi$ is a pseudoscalar light meson called the "Majoron". Reaction I involving the emission of an anti-neu- trino $\bar{v}$ is allowed by the standard electroweak theory, with a predicted halflife of $10^{20} \mathrm{y}$ or more depending on the nucleus. Interest in d.b.d. is mainly related to Reactions II and III which explicitly violate lepton number conservation. Indeed, d.b.d. is probably the most powerful method to test this rule.

Reaction II can occur according to the following scheme: a down quark of a neutron decays emitting an electron $\mathrm{e}^{-}$ and an anti-neutrino which is absorbed by another down quark of a second neutron. This process is possible if the neutrino and the anti-neutrino collide and if they each have a mass, i.e. they are so-called Majorana neutrinos. A second possibility follows from the presence of "right-handed" weak currents. According to the Standard Model, weak currents (analogous to electromagnetic currents in quantum electrodynamics) are "left-handed" and only left-handed neutrinos and right-handed anti-neutrinos can interact. If a small right-handed component is present together with the dominant left-handed one, neutrinoless d.b.d. (Reaction II) could occur.

Double beta decay has only been observed up to now using methods based on geochemical methods and in a few direct experiments. Measured effects have been attributed to the allowed decays but nothing more than stringent lower limits have been set on the forbidden ones. Using these limits it has been possible to set upper limits on the mass of the Majorana neutrino and on the likelihood of the presence of weak righthanded currents.

\section{Multicell and cryogenic detectors}

An experiment using ${ }^{136} \mathrm{Xe}$ is in progress at Gran Sasso: the detector is a multicell proportional chamber filled with isotopically enriched xenon having a total mass of $7 \mathrm{~kg}$. A second experiment based on germanium is being tested and if the results are positive, a large array of ${ }^{76} \mathrm{Ge}$ enriched detectors will be installed in the not-too-distant future.

A very interesting novel technique which exploits cryogenic detectors can 
be used for d.b.d. and for other rare events and is under development in several laboratories around the world. Consider a bolometer: the heat capacity $C$ of a pure dielectric and diamagnetic crystal is given by:

$$
C \sim(T / \theta)^{3}
$$

where $\theta$ and $T$ are the Debye and the absolute temperatures respectively. If $T$ is sufficiently low (a few $\mathrm{mK}$ ), the heat deposited by radiation (ionizing particles, low energy nuclei, etc.) is large enough to increase $T$ by a measurable amount. Very important results have been obtained using the technique and impressive progress was made last year, both in increasing the mass of the bolometers and in improving their energy resolution.

At LNGS, a dilution refrigerator capable of reaching less than $10 \mathrm{mK}$ is in operation. It is the first refrigerator made of specially selected low background materials to be installed in an underground laboratory. Some preliminary but encouraging results have been obtained with a $10.7 \mathrm{~g} \mathrm{Ge}$ crystal; a Te crystal will be used in the near future to search for d.b.d.

\section{Dark Matter}

One of the major problems in astrophysics is the result that the "mechanical" behaviour of galaxies cannot be accounted for in terms of their visible mass, i.e. the mass of the visible stars. The missing mass is usually called "dark matter", the nature of which is at present unknown. The use of bolometers or similar cryogenic devices to detect dark matter is currently under discussion.

\section{High Energy Cosmic Rays}

Many problems remain unsolved in the field of high energy $\left(>10^{12} \mathrm{eV}\right)$ cosmic ray physics. Of special interest is the region of the "knee", at about $10^{6}-10^{7} \mathrm{GeV} /$ nucleon, where the energy spectrum changes slope, becoming steeper as the energy increases. Other features that are of major interest include the existence of point sources, the chemical composition of cosmic rays at high energies, and the presence of high energy neutrinos among primary cosmic rays.

It is well known that high energy cosmic rays interact with the upper atmosphere thereby initiating a cascade or shower of hadrons, photons, electrons, muons, neutrinos, etc. The high energy muons can penetrate a depth equivalent to a thousand metres of water in reaching detectors located deep underground.

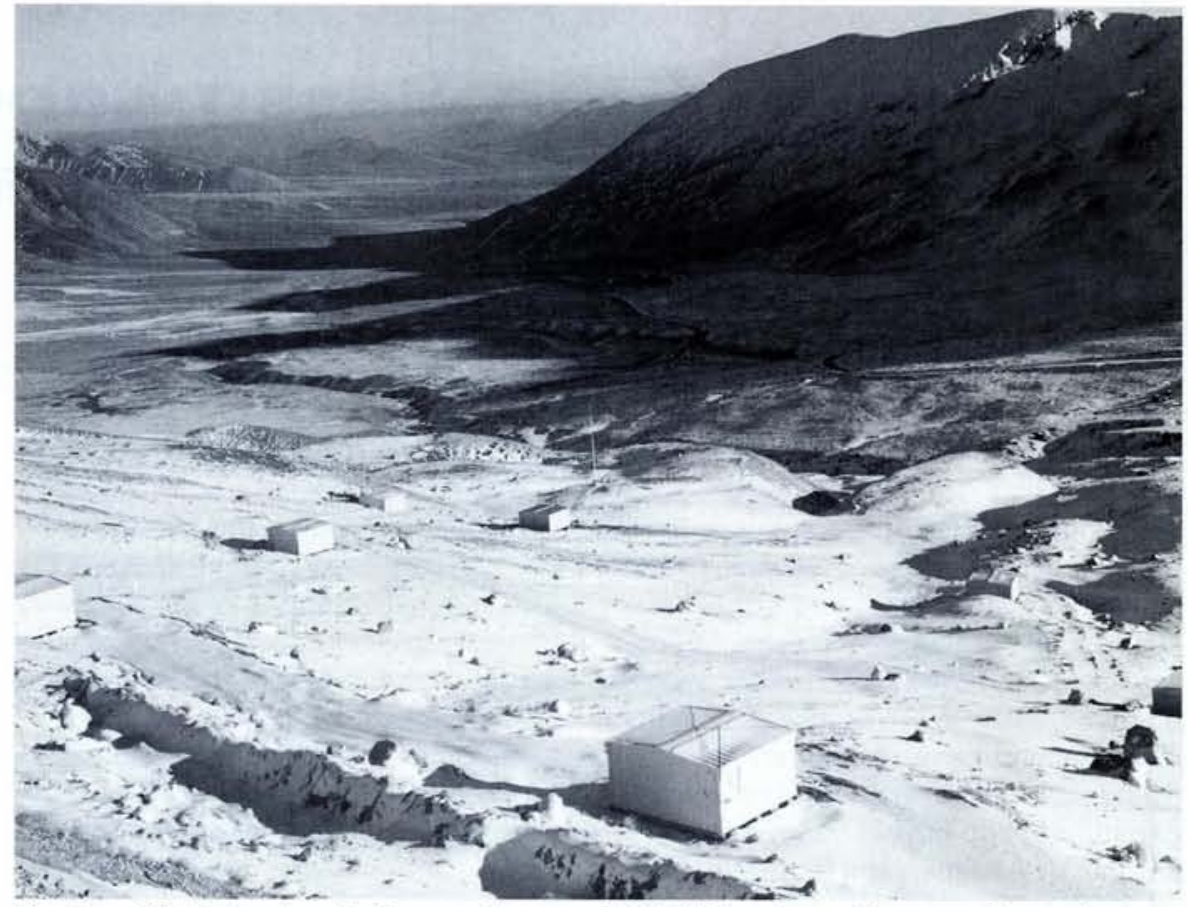

Fig. 1 - The Extensive Air Shower detector (EAS-TOP) at the surface near Campo Imperatore. Each small hut contains $\approx 10 \mathrm{~m}^{2}$ of plastic scintillator. A hadron and muon detector is being installed in the largest building in the foreground.

\section{Surface detectors: EAS-TOP}

At Gran Sasso, cosmic rays are studied both at the surface and deep underground. EAS-TOP, the Extensive Air Shower detector (Fig. 1) located at Campo Imperatore some $2000 \mathrm{~m}$ above sea level consists of an array of 30 detectors spread out over an area of $10^{5} \mathrm{~m}^{2}$. It is used to measure the extensive air showers in the $10^{13}-10^{16}$ $\mathrm{eV}$ range. Data collected over the past two years during assembly of the array have been used to search for point sources such as Cygnus X3. An ultra high energy burst from the Crab Nebula was observed on 23 February 1989 and interesting limits on the activities of various other sources have been settled. The EAS-TOP array will shortly be complemented by a hadron and muon detector located at its centre.

\section{Underground detectors: MACRO, LVD}

Underground in Hall B a large detector called MACRO measures penetrating muons. Designed and constructed by a large Italian-American collaboration to search for astrophysical phenomena, MACRO has a modular structure, being subdivided into six "supermodules" each measuring $12 \times 12 \times 5 \mathrm{~m}^{3}$. The structure is shown in Fig. 2: there are 10 layers of horizontal limited streamer tubes, with read-out in two dimensions, interleaved with passive material. A limited streamer tube is a type of detector that was developed a few years ago at INFN, Frascati. As in proportional and
Geiger counters, particles are detected as a result of the ionisation of a gas in a high electric field. The gas mixture, resistivity and other features of the tube are defined in such a way as to give a spatially limited "streamer" in the tube.

The passive material comprises sheets of CR-39, a track-etch detector. Resembling mica and other materials sensitive to the passage of highly ionizing particles such as fragments of a nucleus, the particles induce structural damage. A subsequent controlled chemical attack reveals the point where each particle crossed a given sheet. Finally, liquid scintillator counters surround the sides of the detector.

The first supermodule is in operation, the remaining five are assembled; data taking with the complete detector will start at the end of 1990.

LVD - the Large Volume Detector - has been specifically designed by another large international collaboration to detect and measure with high efficiency bursts of neutrinos emitted by a collapsing star anywhere in our Galaxy. It comprises approximately 200 modules, each containing eight tonnes of liquid scintillator, surrounded by layers of limited streamer tubes. This detector is however, also capable of investigating other phenomena including penetrating muons and low energy atmospheric neutrinos. It will be installed shortly in Hall $A$ after some technical tests have been completed.

Of special interest are events detected by both EAS-TOP and by the two 
underground detectors. The former measures the "size" of the cosmic ray shower, which is related to the energy of the primary rays, while MACRO and eventually LVD count the number of penetrating muons, which is related to the energy and to the chemical nature of the interacting primary particles. Combining these data it will be possible to determine the chemical composition of cosmic rays at high energies.

It is generally believed that point sources of high energy gamma rays are also sources of neutrinos. These sources can be detected through the observation of muons produced by the interaction of neutrons with rock. Estimates of the muon flux induced by such neutrinos are not very accurate and are on the order of $10^{-16}$ particles $/\left(\mathrm{cm}^{2} \mathrm{~s}\right)$. This very low flux has to be compared to the overwhelming muon flux from standard cosmic ray interactions, and with the unavoidable flux produced by the so-called "atmospheric" neutrinos, i.e. neutrinos produced in air showers.

MACRO and LVD will at least be able to set an accurate limit on the neutrino flux from point sources and, if the present estimates are too pessimistic, allow a signal to be observed.

\section{Magnetic Monopoles}

Many recent cosmological models predict the existence of heavy $\left(10^{16}\right.$ $\mathrm{GeV}$ ) magnetic monopoles. It is also believed that monopole-antimonopole pairs were produced at the very beginning of the Big Bang and that they are now dispersed in the Universe. Their number has not yet been predicted so it is necessary to search for them with large, well shielded detectors.

MACRO with its excellent tracking and timing characteristics and its capability to provide redundant information will allow the clear identification of monopoles no matter whether they are fast (of high energy) or slow.

According to the simple model, the flux of monopoles cannot exceed the so-called "Parker limit" if the galactic magnetic field actually exists. It is therefore essential to reach a detection sensitivity beyond that required by the Parker limit. In five years of operation, a flux of $10^{-16}$ pairs $/\left(\mathrm{cm}^{2} \mathrm{~s} \mathrm{sr}\right)$, well beyond the Parker limit, will be reached.

\section{The Solar Neutrino Problem}

It is generally believed that the Sun produces energy via a chain of thermonuclear reactions and decays which transform four protons in a helium nucleus. These reactions and decays produce neutrinos whose spectrum predicted by the Standard Solar Model is

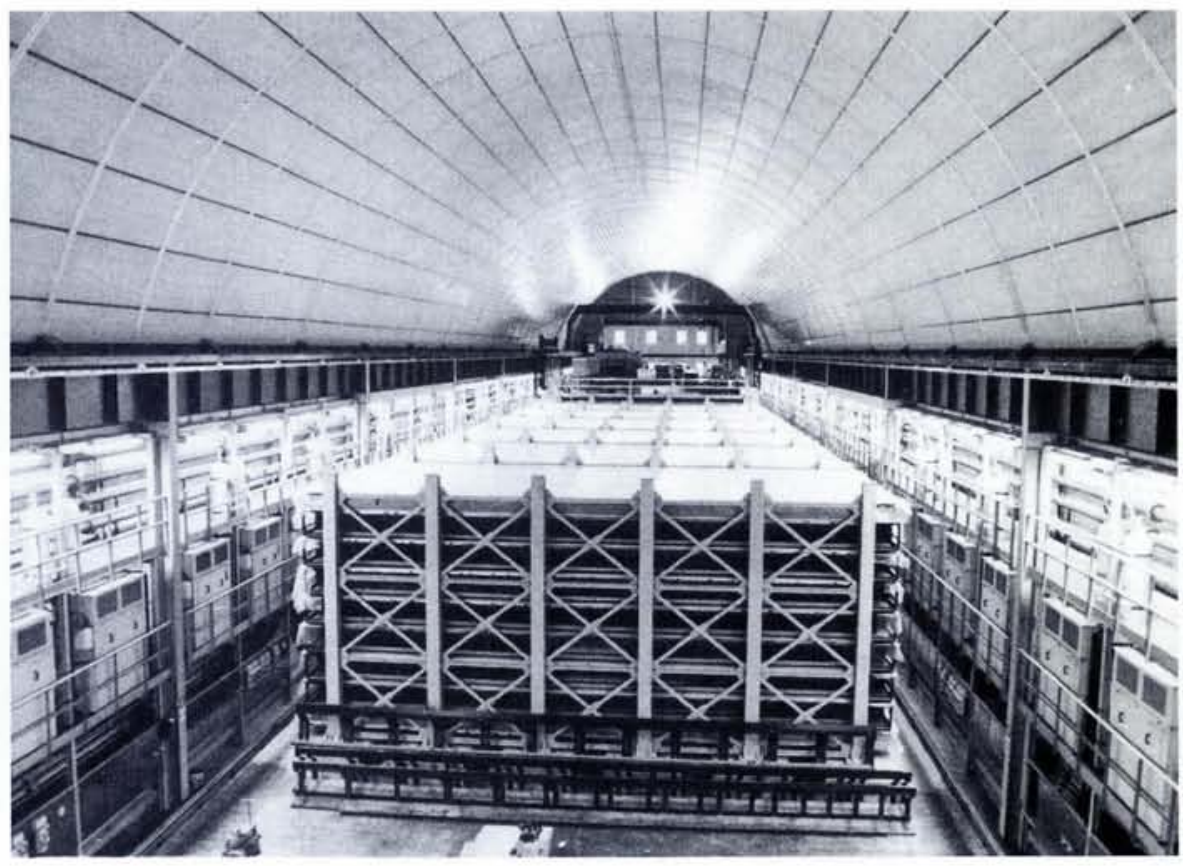

Fig. 2 - The MACRO detector in Hall B. Each of the six $12 \times 12 \times 5 \mathrm{~m}^{3}$ supermodules comprises an array of horizontal streamer tubes interleaved with passive detector material. The walls of the hall are covered with liquid scintillator counters. shown in Fig. 3. Up to now only two experiments have been devoted to solar neutrinos. They are the famous chlorine experiment of R. Davis and, more recently, a Cerenkov detector, based on water, called Kamiokande that was originally designed to search for proton decay.

Both experiments measured the high energy part of the spectrum (the socalled ${ }^{8} \mathrm{~B}$ neutrinos) and both found a flux that was a factor of $\approx 3$ smaller than expected. This is known as the "solar neutrino problem" and the origin of the discrepancy must lie either in the Standard Solar Model, or in the existence of some as yet unidentified features of neutrinos involving, for example, either their oscillations or their magnetic moments.
The need to measure the entire energy spectrum is clear. However, of special interest is the detection of the so-called $\mathrm{p}-\mathrm{p}$ neutrinos (i.e. neutrinos produced by the interactions of protons) because their flux is strictly related to the energy production las opposed to the ${ }^{8} \mathrm{~B}$ neutrinos which are produced in a secondary branch of the fusion chain).

\section{The GALLEX detector}

GALLEX - the Gallium Experiment - is designed by an European collaboration to detect $p-p$ neutrinos using a radiochemical method. The relevant reaction is:

$$
v+{ }^{71} \mathrm{Ga} \rightarrow{ }^{71} \mathrm{Ge}+\mathrm{e}^{-}
$$

${ }^{71} \mathrm{Ge}$ is unstable, decaying back to ${ }^{71} \mathrm{Ga}$ via electron capture with a half-life of 11.4 days.

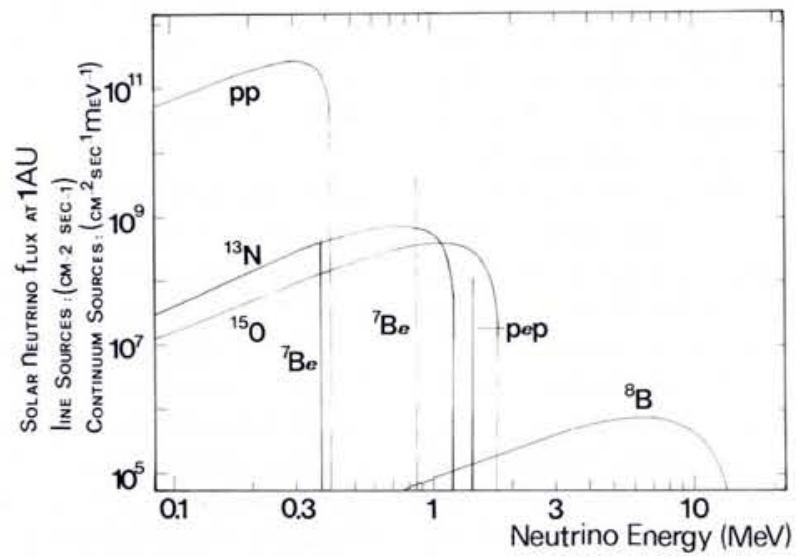

Fig. 3 - The flux of solar neutrinos at one Astronomic Unit from the Sun calculated using the Solar Standard Model. The flux is plotted as a function of the neutrino energy. The various reactions and decays contributing to the flux are indicated for different line and continuum sources (e.g., $p$-p is for the production of neutrons from proton-proton interactions). 


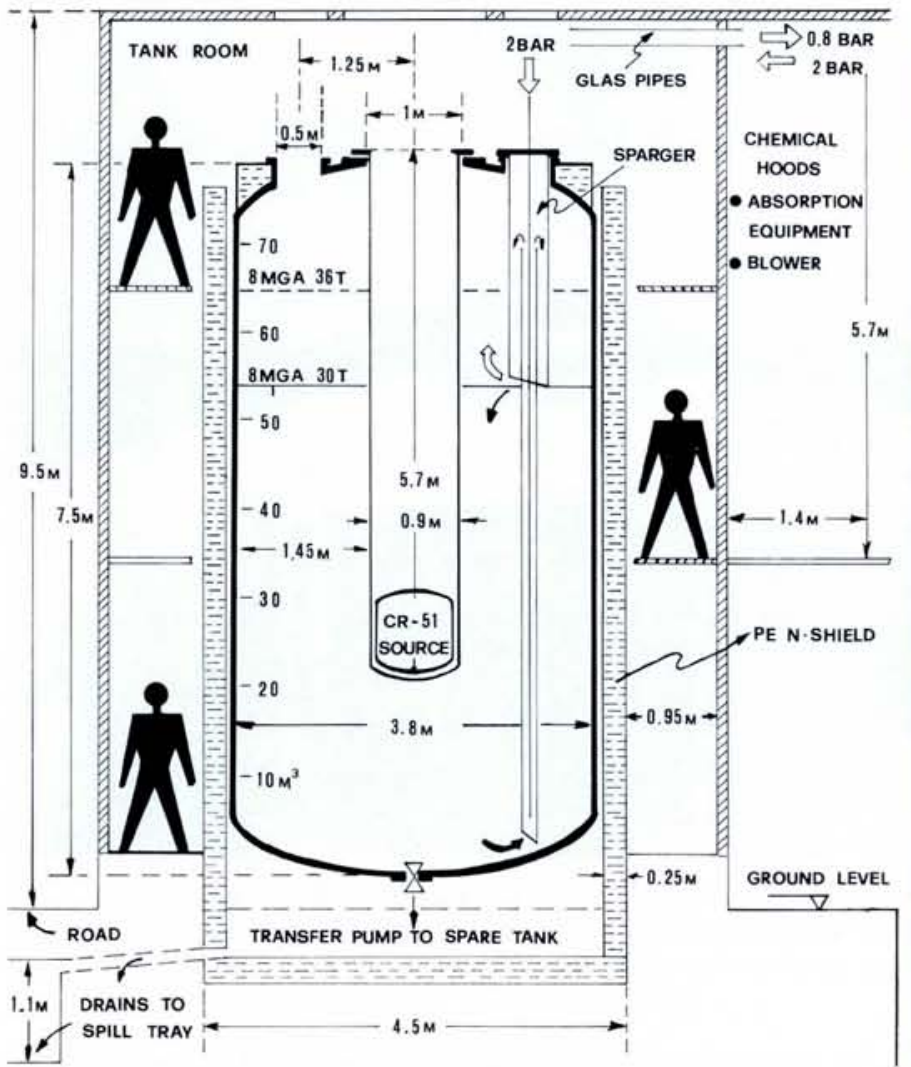

Fig. 4 - The GALLEX detector for p-p neutrinos. Left - a drawing of the detector showing the specially designed, corrosion resistant vessel (made of glass fibre reinforced polyester with an inner lining of polyvinyl fluoride) for the 30 tonnes of gallium as the chloride in an aqueous solution. The vessel will be shielded with a layer of polyethylene or similar material if the neutron flux is too high. A nitrogen purge gas is introduced at a pressure of 2 bar into the upper part of the vessel to extract volatile germanium chloride. The vessel is surrounded by a room that supports walkways. The ${ }^{51} \mathrm{Cr}$ source is for calibration.

Right - a photograph of the two $9.5 \mathrm{~m}$ high GALLEX tanks (one is in reserve).

Thirty tonnes of gallium in a concentrated solution of $\mathrm{GaCl}_{3}-\mathrm{HCl}$ are stored in a large tank installed in Hall A (Fig. 4). The germanium that is produced in the form of volatile $\mathrm{GeCl}_{4}$ will be swept out every two weeks or so by purging with a small amount of nitrogen. $\mathrm{GeCl}_{4}$ will be transformed into $\mathrm{GeH}_{4}$ and used as the filling gas for a specially designed, low-background proportional counter.

According to the Solar Standard Model, one ${ }^{71} \mathrm{Ge}$ atom should be produced daily. This estimate indicates very clearly how delicate and difficult the experiment will be, as well as the number of precautions that will have to be taken to avoid contaminating the solution and the many materials used for the experimental set-up, to avoid any possible source of background radiation. It is also planned to calibrate the experiment using an intense ${ }^{51} \mathrm{Cr}$ source.

The experiment started operation at the end of June 1990 and within one year of data taking, an estimate of the neutrino flux accurate to 10 per cent will be reached if the Standard Solar Model is valid. A result in contradiction with the expected one will necessarily lead to a profound revision of our ideas about the very nature of neutrinos.

\section{ICARUS and BOREX}

Owing to the significance of measurements of solar neutrinos, two other experiments are planned, namely ICARUS and BOREX. The former is a large liquid argon imaging chamber while the sensitive part of BOREX is a boron based liquid scintillator. Prototypes are under construction or being tested to demonstrate the feasibility of these two detectors.

\section{Neutrinos from Collapsing Stars}

A burst of neutrinos emitted by Supernova 1987 was observed for the first time in 1987 by Kamiokande in Japan, IMB in the USA and LSD at Mont Blanc. It gave rise to some of the more interesting results obtained in astrophysics by allowing qualitative confirmation of models describing the collapse of a star. However, more detailed information is needed for a better understanding of this unique phenomenon.

Thanks to the large amount of liquid scintillator (1000 t when completed),
MACRO will be able to detect bursts of low energy neutrinos and anti-neutrinos from collapsing stars. As mentioned earlier, LVD is also specially designed to measure with high efficiency bursts of neutrinos emitted by a collapsing star located anywhere in our Galaxy.

\section{Gravitational Waves}

Very sensitive resonant antennae for gravitational waves have to be operated at very low temperatures in the milli Kelvin regime. They can therefore suffer from noise induced by cosmic rays owing to their interaction, with a large release of energy, with the antennae. Installing these antennae in an underground laboratory such as the Gran Sasso would avoid this problem.

\section{CONCLUSIONS}

The Gran Sasso Laboratory has been well received by the scientific community and has already met with considerable success.

A plan to provide two additional underground halls (dedicated to experiments based on cryogenic techniques and to those needing a low radioactive background), an independent access tunnel, new surface facilities and an improved infrastructure has been approved by the Italian Parliament. Its final approval, which is expected shortly, will enhance the Laboratory's scientific activity involving several fundamental and important aspects of astrophysics and cosmology. 